\title{
Spectral element method for wave propagation on irregular domains
}

\author{
YAN HUI GENG, GUO LIANG QIN* and JIA ZHONG ZHANG
}

School of Energy and Power Engineering, Xi'an Jiaotong University, Xi'an 710049, China

e-mail: ling.zhi.geng@stu.xjtu.edu.cn; glqin@mail.xjtu.edu.cn; jzzhang@mail.xjtu.edu.cn

MS received 31 December 2016; revised 7 May 2017; accepted 28 July 2017; published online 14 March 2018

\begin{abstract}
A spectral element approximation of acoustic propagation problems combined with a new mapping method on irregular domains is proposed. Following this method, the Gauss-Lobatto-Chebyshev nodes in the standard space are applied to the spectral element method (SEM). The nodes in the physical space are mapped according to the length scale of the beeline segment or the curve segment. Using the Bubnov-Galerkin method, some acoustic problems with two kinds of irregular domains are simulated in detail. First, the basic problem with analytical solution is analysed numerically. Numerical results show that the SEM integrated with the lengthscale method has the same precision as the isoparametric SEM. Also, it can save nearly half of the time cost. Additionally, the acoustic propagations with inlet flow are simulated numerically. All the results indicate that the SEM integrated with the length-scale method has the ability to simulate the acoustic problems with irregular domains. It is shown that the mapping method maintains the curve edges and provides a useful alternative for isoparametric element, which represents a curved edge with a straight edge.
\end{abstract}

Keywords. Spectral element method; curved quadrilateral element; isoparametric element; Chebyshev polynomial; mapping method.

\section{Introduction}

Arbitrary geometry domain is of prime interest in many fields of engineering such as acoustics, soil-structure interaction, fluid-structure interaction, the aircraft, automotive, shipbuilding and others. The domains are desired to be smooth and fair, but are always irregularly shaped. In numerical methods, the meshing of the irregular domains remains one of the most cumbersome and important steps in the entire solution process. To deal with problems with irregular domains, some methods have been proposed to overcome this problem, such as meshless local strong form method [9], the Chebyshev tau meshless method based on the integration-differentiation (CMMID) [19], direct expansion method of boundary condition (DEMBC) [24], boundary element method (BEM) [10], the finite element method (FEM) based on the triangular elements [23], the level-set function [21], the fractional step projection scheme [4], the Coons method, the Laplace method and boundary-blending method [3], isoparametric method [13], etc.

Spectral element method (SEM) invented by Patera [16] in 1984 is successfully used nowadays for widely diverse applications, such as fluid dynamics, wave propagation, solid and structural analysis, and so on [22]. The SEM may be viewed as one case of the Bubnov-Galerkin method. In

*For correspondence the Chebyshev SEM, the solution is approximated by means of a truncated Chebyshev series; the Chebyshev polynomials are used as the test function. It is a combination of spectral method and FEM. Its principal advantage relies on its superior rate of convergence (spectral accuracy) for sufficiently smooth functions and its flexibility to describe complex geometries despite the computational costs associated with constructing an appropriate solution grid. However, the main limitation of SEMs is that the geometry of the domain in which the problem is to be solved must be regular enough to allow the use of an appropriate orthonormal basis to expand the full set of possible solutions. This inability to handle irregularly shaped domains is the main reason why SEM has had limited use in many engineering problems.

There are many works in the existing literature in which higher order spectral elements handling arbitrary geometry are demonstrated. Mengaldo et al [11] improved the robustness of high-order SEM using two dealiasing techniques for solving the incompressible flow around the airfoil NACA0012. However, the triangular element is a more common choice. Giraldo and Warburton [6] proposed the diagonal-mass-matrix triangularspectral-element method, which was applicable for arbitrary regions, and was applied for the shallow-water equations inside a sphere [5]. Pasquetti and Rapetti [15] compared the SEMs based on the triangular elements and quadrilateral elements, and the triangular element was 

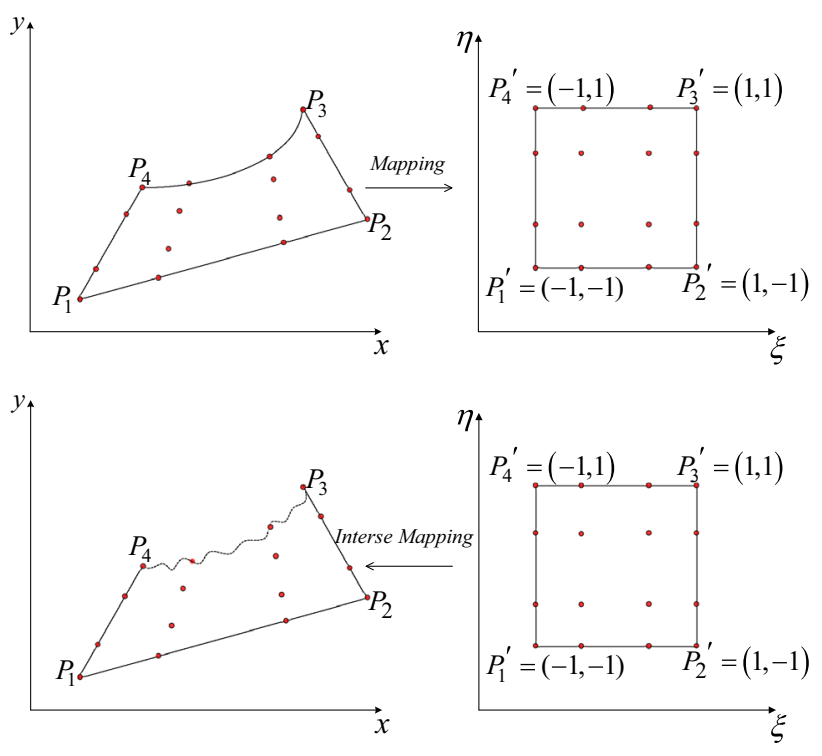

Figure 1. Sketch illustrating the irreversibility of the mapping.

found to give a higher flexibility. Mercerat et al [12] simulated a two-dimensional elastic wave. Also the incompressible Navier-Stokes equation could be discretized by the triangular element [1, 17]. All these research works were carried out on the straight-edge triangular element, and the numerical accuracy was greatly reduced if the curved boundary was treated as a straight edge. If the domain is not polygonal, the accuracy of SEM quickly deteriorates if curved elements are not implemented [14]. Hence Pasquetti [14] proposed a Gauss-Fekete SEM on the curved triangular elements. The author was motivated by Pasquetti [14] and tried the SEM on the curved quadrilateral elements in this paper.

The rest of the paper is organized as follows. In section 2, the "length-scale method" is proposed for the mapping from the irregular domain/element to the standard element. The basic formulas of acoustic model are given and the numerical procedures for applying SEM to acoustic problems are detailed in section 3. Section 4 gives some numerical examples on the irregular domains, including trapezoidal domain and rectangular domain pierced by a circular hole. A conclusion is presented in section 5 .

\section{Length-scale method}

In many computations, Gauss integral always needs to be carried out on the irregular elements and sometimes it is very difficult to be calculated. Therefore, the standard element is usually used to resolve this issue. Hence, the conversion of the irregular element to the standard element is particularly important. The transforming matrix, known as the Jacobian matrix, is defined as

$$
J=\frac{\partial(x, y)}{\partial(\xi, \eta)}=\left[\begin{array}{ll}
\frac{\partial x}{\partial \xi} & \frac{\partial y}{\partial \xi} \\
\frac{\partial x}{\partial \eta} & \frac{\partial y}{\partial \eta}
\end{array}\right]
$$

where $x, y$ are the Cartesian coordinates of the physical space and $\xi, \eta$ the local coordinates of the standard space. In the Galerkin method, $|J|$ (the determinant of the Jacobian matrix) always stays in the form of the product with other items in the integral sign. It is always necessary to use numerical integral and/or FEM/SEM discretization because the integration cannot be carried analytically.

The transformation of the physical space and the standard space is a kind of mapping. Most existing mapping methods that are suitable for SEM on irregular domains are wonderful in the straight edges elements. However, they have their shortcomings when they are applied to curved edges even if the isoparametric mapping is usually substituted into the affine. Hence, the transformation from the physical element to the standard element is defined as the mapping. Thus the transformation from the standard element to the physical element is defined as the inverse mapping. The standard element nodes are deployed at positions $\xi_{i}$ for $i=1, \ldots, m+1$ along the $\xi$ axis and $\eta_{j}$ for $j=1, \ldots, n+1$ along the $\eta$ axis. The corresponding positions along the $x, y$ axes arise from the inverse mapping, and vice versa. That is to say, the mapping is
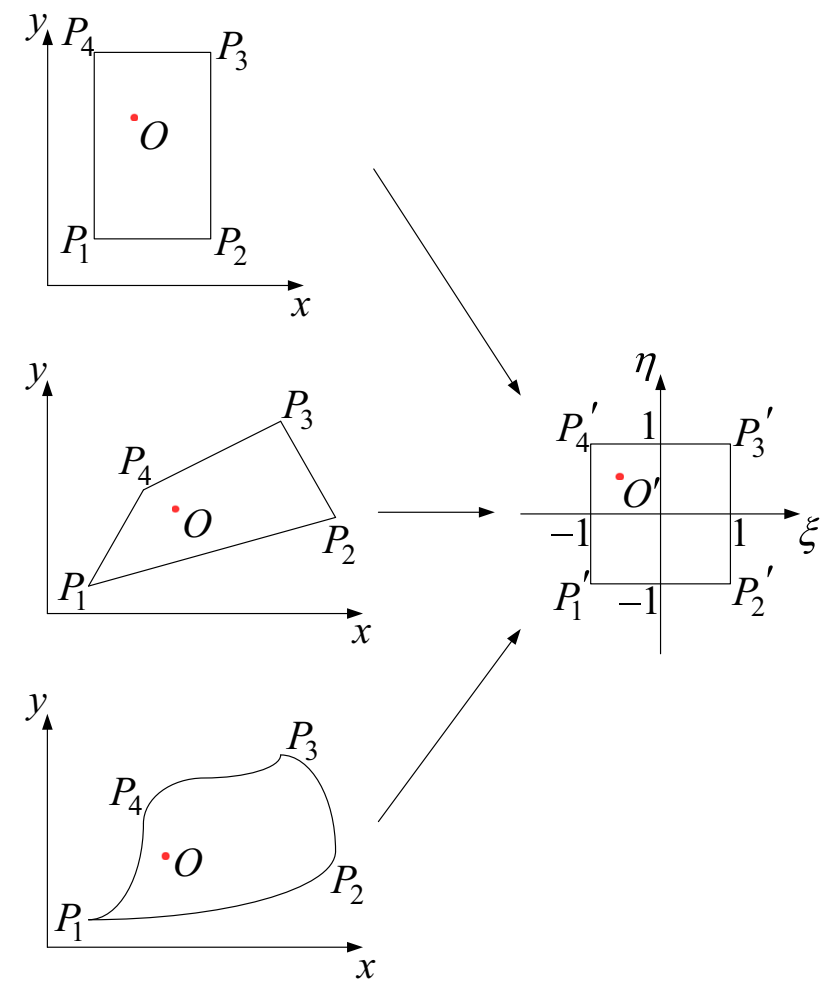

Figure 2. Arbitrary quadrilateral converted to a standard element. 

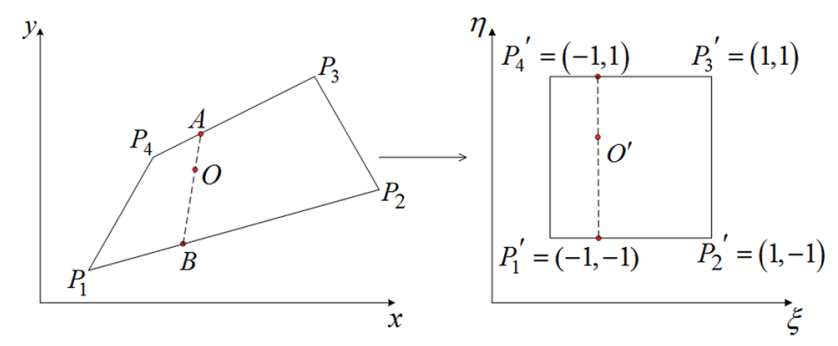

Figure 3. Length-scale method-straight-sided quadrilateral.

reversible at the interpolation nodes. However, the mapping is not invertible on the curved segments except for interpolation nodes. A sketch illustrating the irreversibility of the mapping is given in figure 1 .

In view of this shortcoming, the length-scale method based on the geometry of the curves or beelines is proposed in this paper. The basic principle of length-scale method is that the curves/beelines are divided by the interpolation nodes by measuring the length of the curves/beelines. Thus the mapping relationship between the physical space and the standard space is established through some algebraic transformations. This mapping is invertible elsewhere because the length of curves/beelines is an explicit exact function of $(x, y)$.

Let $\Omega$ be a two-dimensional domain and consider a mesh $T_{h}$ of $\Omega$ composed of simplices (i.e., Cartesian, cylindrical, spherical, ...). As is well known, if $\Omega$ is not polygonal then the mesh $T_{h}$ is no-longer satisfactory in a high-order approximation. What is considered in this paper is that $e \in T_{h}$ is a quadrilateral. Arbitrary quadrilateral being converted to a standard square is shown in figure 2, including rectangle, arbitrary straight-sided quadrilateral and curve-sided quadrilateral. The main contents of this paper focus on the latter two cases.

\section{1) Quadrilateral with straight edges}

The procedure of length-scale method for straight-sided quadrilaterals is presented in the schematic diagram in figure 3. First, seek points $A$ and $B$, respectively, on the beeline segments $P_{3} P_{4}$ and $P_{1} P_{2}$ that meet

$$
\left\{\begin{array}{l}
\frac{P_{4} A}{P_{4} P_{3}}=\frac{\xi+1}{2} \\
\frac{P_{1} B}{P_{1} P_{2}}=\frac{\xi+1}{2}
\end{array}\right.
$$

Then seek point $O$ on the beeline segment $A B$ that meets

$$
\frac{B O}{B A}=\frac{\eta+1}{2}
$$

The point $O(x, y)$ in the physical space corresponds to the point $O^{\prime}(\xi, \eta)$ in the standard space. Through simultaneous equations (2) and (3), the mapping relationship between the physical space and the standard space is established, as follows:

$$
\left\{\begin{array}{l}
x=a(\eta+1)(\xi+1)+b(\eta+1)+c(\xi+1)+d \\
y=e(\eta+1)(\xi+1)+f(\eta+1)+g(\xi+1)+h
\end{array}\right.
$$

The Jacobian matrix is easily derived as

$$
J=\left[\begin{array}{ll}
a(\eta+1)+c & e(\eta+1)+g \\
a(\xi+1)+b & e(\xi+1)+f
\end{array}\right]
$$

where

$$
\begin{cases}a=\frac{x_{3}-x_{4}-x_{2}+x_{1}}{4}, & b=\frac{x_{4}-x_{1}}{2}, \\ c=\frac{x_{2}-x_{1}}{2}, d=x_{1} & \\ e=\frac{y_{3}-y_{4}-y_{2}+y_{1}}{4}, & f=\frac{y_{4}-y_{1}}{2}, \\ g=\frac{y_{2}-y_{1}}{2}, h=y_{1} & \end{cases}
$$

It is noteworthy that Eqs. (4) and (5) are the same as Eqs. (4.10.1) and (4.10.4) in reference [18]. Thus it is approved that the length-scale method is feasible in principle.

\section{2) Quadrilateral with curved edges}

As long as the length of the curves can be provided with a specific expression, the mapping relationship between the curved quadrilateral and the standard element can be obtained by the length-scale method. If $e \in T_{h}$ is a curved quadrilateral, it is assumed to be in the simple case where two of the vertices, say $P_{1}$ and $P_{2}$, are in $\Omega$ whereas the two others, $P_{3}$ and $P_{4}$, are on boundary, and that the segment $P_{3} P_{4}$ is a circular arc $\left(\overparen{P_{3} P_{4}}\right)$ whereas $P_{1} P_{4}, P_{2} P_{3}, P_{3} P_{4}$ remain straight, see figure 4 . This may not be sufficient to address some more complex situations where the whole mesh should be deformed and not only the boundary cells. As shown in figure $4, \Theta$ is the centre of a circle of radius $R=\Theta P_{4}=\Theta P_{3}$ and $\Theta C$ is parallel to the $x$ axis; $c_{1}$ and $c_{2}$ are the radians of $\angle C \Theta P_{4}$ and $\angle C \Theta P_{3}$, respectively. All of these are known. First, seek the point $A$ on the arc segment $\overparen{P_{4} P_{3}}$ and the point $B$ on the beeline segment $P_{1} P_{2}$ that meet

$$
\left\{\begin{array}{l}
\frac{\overparen{P_{4} A}}{\overparen{P_{4} P_{3}}}=\frac{\xi+1}{2} \\
\frac{P_{1} B}{P_{1} P_{2}}=\frac{\xi+1}{2}
\end{array}\right.
$$

Then seek the point $O(x, y)$ on the beeline segment $A B$ that meets

$$
\frac{B O}{B A}=\frac{\eta+1}{2} .
$$

Through simultaneous equations (7) and (8), thus, the mapping relationship between the physical space and the standard space is established, ${ }^{1}$ as follows:

\footnotetext{
${ }^{1} \mathrm{~A}$ brief clarification in obtaining Eq. (9) is given in Appendix A.
} 

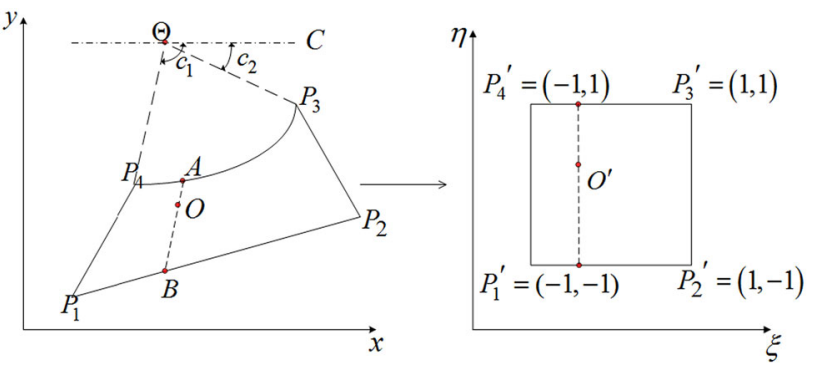

Figure 4. Length-scale method—curved quadrilateral.

$$
\left\{\begin{array}{c}
x=\frac{\eta+1}{2}\{R \cos [a(\xi+1)+b]-c \xi+d\}+c \xi+e \\
y=\frac{\eta+1}{2}\{R \sin [a(\xi+1)+b]-f \xi+g\}+f \xi+h
\end{array} .\right.
$$

Hence, the Jacobian matrix can be derived as

$$
J=\left[\begin{array}{ll}
J_{11} & J_{12} \\
J_{21} & J_{22}
\end{array}\right]
$$

where

$$
\begin{gathered}
J_{11}=\frac{\eta+1}{2}\{-R a \sin [a(\xi+1)+b]-c\}+\frac{c}{2} \\
J_{12}=\frac{\eta+1}{2}\{R a \cos [a(\xi+1)+b]-f\}+\frac{f}{2} \\
J_{21}=\frac{1}{2}\{R \cos [a(\xi+1)+b]-c \xi+d\} \\
\left\{\begin{array}{l}
J_{22}=\frac{1}{2}\{R \sin [a(\xi+1)+b]-f \xi+g\} \\
a=\frac{c_{2}-c_{1}}{2}, \quad b=c_{1}, \\
f=\frac{y_{2}-y_{1}}{2}, \quad g=x_{0}-x_{1}-\frac{x_{2}-x_{1}}{2}, \quad e=\frac{x_{2}+x_{1}}{2}
\end{array} .\right.
\end{gathered}
$$

\section{Numerical method}

\subsection{Governing equations}

The aerodynamic acoustics study focusses on the capture of the acoustic vibration-induced pressure distribution. In this paper, the sound wave in the fluid without absorption, attenuation or viscidity is studied. It is assumed that a mass source of magnitude $Q\left(\mathrm{~kg} /\left(\mathrm{m}^{3} \mathrm{~s}\right)\right)$ exists in the domain, and a force $F\left(\mathrm{~N} / \mathrm{m}^{3}\right)$ acts on the fluid microelement. According to the method of Goldstein [7], the governing equation of the sound pressure $p$ is obtained as

$$
\begin{aligned}
& \frac{\partial^{2} p}{\partial t^{2}}+2 c_{0} M a_{x} \frac{\partial^{2} p}{\partial x \partial t}+2 c_{0} M a_{y} \frac{\partial^{2} p}{\partial y \partial t}+2 c_{0}^{2} M a_{x} M a_{y} \frac{\partial^{2} p}{\partial x \partial y} \\
& -c_{0}^{2}\left[\left(1-M a_{x}^{2}\right) \frac{\partial^{2} p}{\partial x^{2}}+\left(1-M a_{y}^{2}\right) \frac{\partial^{2} p}{\partial y^{2}}\right] \\
& +c_{0}\left(\frac{\partial M a_{x}}{\partial t}+c_{0} M a_{x} \frac{\partial M a_{x}}{\partial x}+M a_{y} \frac{\partial M a_{x}}{\partial y}\right) \frac{\partial p}{\partial x} \\
& +c_{0}\left(\frac{\partial M a_{y}}{\partial t}+c_{0} M a_{x} \frac{\partial M a_{y}}{\partial x}+M a_{y} \frac{\partial M a_{y}}{\partial y}\right) \frac{\partial p}{\partial y}=f
\end{aligned}
$$

where $c_{0}$ is the local sound velocity; $M a_{x}=u_{01} / c_{0}$ and $M a_{y}=u_{02} / c_{0}$ are the components of Mach number $M a$ along $x$ and $y$ directions, respectively, the subscript " 0 " denotes the physical quantities without sound field, the subscripts " 1 ", " 2 " denote the indices of velocity in Cartesian coordinate system; $f=c_{0}^{2}\left(\frac{D Q}{\partial t}-\frac{\partial^{2} \varepsilon_{i j}}{\partial y_{i} \partial y_{j}}-\frac{\partial F_{i}}{\partial y_{i}}\right)$ is the summation of the source terms, where $D / D t$ is the material derivative defined as $D / D t=\partial / \partial t+u_{0 i} \partial / \partial y_{i}$, $\varepsilon_{i j}=\mu\left[\partial u_{i} / \partial y_{j}+\partial u_{j} / \partial y_{i}-(2 / 3) \delta_{i j} \partial u_{k} / \partial y_{k}\right]$ is the viscous stress tensor and $\delta_{i j}$ is the Kronecker delta. As for boundary conditions, we restrict ourselves to the study of the Dirichlet boundaries, the Neumann boundaries and the nonreflecting boundaries. In this paper, the $\mathrm{BGH}$ absorbing boundary conditions [2] are applied to the wave propagation problems with flow background.

\subsection{Variational formulation}

The variational problem of Eq. (12) is to find a function $u \in H^{1}$ such that

$$
B(p, v)+T_{\Gamma_{N R}}(p, v)=S(f, v)+T_{\Gamma_{N}}(v), \quad \forall v \in H_{0}^{1}
$$

where $T_{\Gamma_{N R}}(p, v)$ is the term induced by non-reflecting boundaries and $T_{\Gamma_{N}}(p, v)$ is the term induced by Neumann boundaries. The expansion of each term in Eq. (17) in the two-dimensional Cartesian coordinate system is

$$
\begin{aligned}
& B(p, v)=\iint\left[\begin{array}{r}
\frac{\partial^{2} p}{\partial t^{2}}+2 c_{0} M a_{x} \frac{\partial^{2} p}{\partial x \partial t}+2 c_{0} M a_{y} \frac{\partial^{2} p}{\partial y \partial t}+ \\
\left.2 c_{0}^{2} M a_{x} M a_{y} \frac{\partial^{2} p}{\partial x \partial y}\right]
\end{array}\right] v d x d y \\
& +\iint c_{0}^{2}\left[\left(1-M a_{x}^{2}\right) \frac{\partial p \partial}{\partial x} \frac{\partial v}{\partial x}+\left(1-M a_{y}^{2}\right) \frac{\partial p}{\partial y} \frac{\partial v}{\partial y}\right] d x d y \\
& +\iint\left[\begin{array}{c}
c_{0}\left(\frac{\partial M a_{x}}{\partial t}+c_{0} M a_{x} \frac{\partial M a_{x}}{\partial x}+M a_{y} \frac{\partial M a_{x}}{\partial y}\right) \frac{\partial p^{\prime}}{\partial x}+ \\
c_{0}\left(\frac{\partial M a_{y}}{\partial t}+c_{0} M a_{x} \frac{\partial M a_{y}}{\partial x}+M a_{y} \frac{\partial M a_{y}}{\partial y}\right) \frac{\partial p^{\prime}}{\partial y}
\end{array}\right] v d x d y
\end{aligned}
$$

$$
S(f, v)=\iint f v d x d y
$$




$$
\begin{aligned}
T_{\Gamma_{N R}}(p, v)= & -c_{0}^{2} \int_{\Gamma_{N R}}\left(1-M a_{x}^{2}\right) \frac{\partial p}{\partial x} n_{x} v d s \\
& -c_{0}^{2} \int_{\Gamma_{N R}}\left(1-M a_{y}^{2}\right) \frac{\partial p}{\partial y} n_{y} v d s \\
T_{\Gamma_{N}}(p, v)= & c_{0}^{2} \int_{\Gamma_{N}}\left(1-M a_{x}^{2}\right) \frac{\partial p}{\partial x} n_{x} v d s \\
& +c_{0}^{2} \int_{\Gamma_{N}}\left(1-M a_{y}^{2}\right) \frac{\partial p}{\partial y} n_{y} v d s
\end{aligned}
$$

where $\mathbf{n}=\left(n_{x}, n_{y}\right)$ is the unit out normal vector to the boundaries.

\subsection{Space discretization}

The Chebyshev spectral element approximation will be used on each element after discretizing the computational domain into $\mathrm{Ne}$ non-overlapping elements. Each element is transformed into the standard element by the length-scale method detailed in section 2. On each element, all functions can be written as

$$
\begin{aligned}
& p_{h}=\sum_{j=0}^{N_{x}} \sum_{k=0}^{N_{y}} p_{j k} N_{j k}(\xi, \eta) \\
& v_{h}=\sum_{p=0}^{N_{x}} \sum_{q=0}^{N_{y}} v_{p q} N_{p q}(\xi, \eta)
\end{aligned}
$$

where $N_{x}, N_{y}$ are the orders of the Chebyshev polynomial in the $x, y$ directions, respectively, and $N_{j k}(-$ $\xi, \eta)=h_{j}(\xi) h_{k}(\eta)$ is the basis function; $h_{j}(\xi)$ and $h_{k}(\eta)$ are the Lagrangian interpolation functions satisfying $h_{j}\left(\phi_{m}\right)=\delta_{j m}$. Substituting Eqs. (22) and (23) into Eq. (17) yields

$$
\begin{array}{r}
B_{e}^{V}\left(N_{j k} p_{j k}, N_{p q}\right)=M_{e}^{V} \ddot{p}_{h}+C_{e}^{V} \dot{p}_{h}+K_{e}^{V} p_{h} \\
S_{e}^{V}\left(N_{p q}\right)=\sum_{j=0}^{N_{x}} \sum_{k=0}^{N_{y}} f_{j k} \iint N_{j k} N_{p q} d x d y \\
=\sum_{j=0}^{N_{x}} \sum_{k=0}^{N_{y}} f_{j k} \sum_{m=0}^{N_{x}} \sum_{n=0}^{N_{y}}|J|_{m n} B_{j p m}^{\xi} B_{k q n}^{\eta}
\end{array}
$$

where the superscript " $V$ " denotes the terms calculated on the domain, $M_{e}^{V}$ the quality matrix, $C_{e}^{V}$ the damping matrix and $K_{e}^{V}$ the stiffness matrix:

$$
M_{e}^{V}=\sum_{m=0}^{N_{x}} \sum_{n=0}^{N_{y}}|J|_{m n} B_{j p m}^{\xi} B_{k q n}^{\eta}
$$

$$
\begin{aligned}
C_{e}^{V}= & 2 c_{0} \sum_{r=0}^{N_{x}} \sum_{s=0}^{N_{y}} \sum_{m=0}^{N_{x}} \sum_{n=0}^{N_{y}}\left(M a_{x}\right)_{r s} \nabla_{x, j k m n} B_{r p m}^{\xi} B_{s q n}^{\eta} \\
& +2 c_{0} \sum_{r=0}^{N_{x}} \sum_{s=0}^{N_{y}} \sum_{m=0}^{N_{x}} \sum_{n=0}^{N_{y}}\left(M a_{y}\right)_{r s} \nabla_{y, j k m n} B_{r p m}^{\xi} B_{s q n}^{\eta} \\
K_{e}^{V}= & 2 c_{0}^{2} \sum_{c=0}^{N_{x}} \sum_{d=0}^{N_{y}}\left(M a_{x} M a_{y}\right)_{c d} C_{c j p}^{\xi} C_{d k q}^{\eta} \\
& +c_{0}^{2} \sum_{m=0}^{N_{x}} \sum_{n=0}^{N_{y}} \sum_{r=0}^{N_{x}} \sum_{s=0}^{N_{y}} \sum_{a=0}^{N_{x}} \sum_{b=0}^{N_{y}} \sum_{c=0}^{N_{x}} \sum_{d=0}^{N_{y}} \\
& \times\left(1-M a_{x}^{2}\right)_{c d} \frac{1}{|J|_{m n}} \nabla_{x, j k r s} \nabla_{x, p q a b} B_{c m r a}^{\xi} B_{d n s b}^{\eta} \\
& +c_{0}^{2} \sum_{m=0}^{N_{x}} \sum_{n=0}^{N_{y}} \sum_{r=0}^{N_{x}} \sum_{s=0}^{N_{y}} \sum_{a=0}^{N_{x}} \sum_{b=0}^{N_{y}} \sum_{c=0}^{N_{x}} \sum_{d=0}^{N_{y}} \\
& \times\left(1-M a_{y}^{2}\right)_{c d} \frac{1}{|J|_{m n}} \nabla_{y, j k r s} \nabla_{y, p q a b} B_{c m r a}^{\xi} B_{d n s b}^{\eta} \\
& +c_{0} \sum_{c=0}^{N_{x}} \sum_{d=0}^{N_{y}} \sum_{r=0}^{N_{x}} \sum_{s=0}^{N_{y}}\left(\frac{\partial M a_{x}}{\partial t}+c_{0} M a_{x} \frac{\partial M a_{x}}{\partial x}\right. \\
& \left.+M a_{y} \frac{\partial M a_{x}}{\partial y}\right)_{c d}^{\nabla_{x, j k r s}} B_{c r p}^{\xi} B_{d s q}^{\eta} \\
+ & c_{0} \sum_{c=0}^{N_{x}} \sum_{d=0}^{N_{y}} \sum_{r=0}^{N_{x}} \sum_{s=0}^{N_{y}}\left(\frac{\partial M a_{y}}{\partial t}+c_{0} M a_{x} \frac{\partial M a_{y}}{\partial x}\right. \\
& \left.M a_{y} \frac{\partial a_{y}}{\partial y}\right)_{c d} \nabla_{y, j k r s} B_{c r p}^{\xi} B_{d s q}^{\eta}
\end{aligned}
$$

where

$$
\begin{gathered}
C_{j p m}^{\phi}=\int_{-1}^{1} h_{j}(\phi) \frac{\partial h_{p}(\phi)}{\partial \phi} h_{m}(\phi) d \phi \\
B_{j p m}^{\phi}=\int_{-1}^{1} h_{j}(\phi) h_{p}(\phi) h_{m}(\phi) d \phi \\
B_{j p m r}^{\phi}=\int_{-1}^{1} h_{j}(\phi) h_{p}(\phi) h_{m}(\phi) h_{r}(\phi) d \phi \\
\nabla_{x, j k m n}=\left(\frac{\partial y}{\partial \eta} \frac{\partial N_{j k}}{\partial \xi}-\frac{\partial y}{\partial \xi} \frac{\partial N_{j k}}{\partial \eta}\right)_{m n} \\
=\left(\frac{\partial y}{\partial \eta}\right)_{m n} D_{j m} \delta_{k n}-\left(\frac{\partial y}{\partial \xi}\right)_{m n} \delta_{j m} D_{k n} \\
\nabla_{y, j k m n}=\left(-\frac{\partial x}{\partial \eta} \frac{\partial N_{j k}}{\partial \xi}+\frac{\partial x}{\partial \xi} \frac{\partial N_{j k}}{\partial \eta}\right)_{m n} \\
=-\left(\frac{\partial x}{\partial \eta}\right)_{m n} D_{j m} \delta_{k n}+\left(\frac{\partial x}{\partial \xi}\right)_{m n} \delta_{j m} D_{k n}
\end{gathered}
$$


where $j, m=0 \sim N_{x} ; k, n=0 \sim N_{y} ; D_{j m}, D_{k n}$ are the differential matrices

$$
D_{j m}=\left.\frac{\partial h_{j}(\phi)}{\partial \phi}\right|_{\phi=\phi_{m}} .
$$

The discretizations of Eqs. (16) and (17) have different expressions in different shaped domains. For the domains discussed in section 4 , the discrete formulas are exhibited in the following. For Case 2 in section 4.1, the damping matrices and the stiffness matrices induced by the absorbing boundary conditions are

$$
\begin{gathered}
C_{e}^{\Gamma}=c_{0} \sum_{a=0}^{N_{x}} \sum_{m=0}^{N_{x}}(1-\mathbf{n} \cdot \mathbf{M a})_{a b} \\
\left(\sqrt{\left(\frac{\partial x}{\partial \xi}\right)^{2}+\left(\frac{\partial y}{\partial \xi}\right)^{2}}\right) B_{j a p m}^{\xi} \\
\left(j=0 \sim N_{x}, b=n=k=q=0\right)
\end{gathered}
$$

where $\mathbf{M a}=\left(M a_{x}, M a_{y}\right)$ is defined as a vector composed of two numbers $M a_{x}$ and $M a_{y}$.

$$
\begin{gathered}
K_{e}^{\Gamma_{y}}=c_{0}^{2} \sum_{a=0}^{N_{x}} \sum_{m=0}^{N_{x}} \sum_{r=0}^{N_{x}}[(1-\mathbf{n} \cdot \mathbf{M a})|\mathbf{n M a}|]_{a b} \frac{1}{|J|_{m n}} \nabla_{y, j k m n} \\
\left(\sqrt{\left(\frac{\partial x}{\partial \xi}\right)^{2}+\left(\frac{\partial y}{\partial \xi}\right)^{2}}\right)_{r s} B_{a m p r, b=n=s=k=q=0}^{i} \\
K_{e}^{\Gamma_{x}}=c_{0}^{2} \sum_{a=0}^{N_{x}} \sum_{m=0}^{N_{x}} \sum_{r=0}^{N_{x}}[(1-\mathbf{n} \cdot \mathbf{M a})|\mathbf{n} \times \mathbf{M a}|]_{a b} \frac{1}{|J|_{m n}} \nabla_{x, j k m n} \\
\left(\sqrt{\left(\frac{\partial x}{\partial \xi}\right)^{2}+\left(\frac{\partial y}{\partial \xi}\right)^{2}}\right)_{r s}^{i} B_{a m p r, b=n=s=k=q=0}
\end{gathered}
$$

where the superscripts " $\Gamma_{x}, \Gamma_{y}$ " denote the boundaries along the $x, y$ axes, respectively.

For Case 2 in section 4.2, the damping matrices and the stiffness matrices induced by the absorbing boundary conditions are

$$
\begin{gathered}
C_{e}^{\Gamma_{y}}=c_{0} \sum_{b=0}^{N_{y}} \sum_{n=0}^{N_{y}}(1-\mathbf{n} \cdot \mathbf{M a})_{a b}\left(\sqrt{\left(\frac{\partial x}{\partial \eta}\right)^{2}+\left(\frac{\partial y}{\partial \eta}\right)^{2}}\right)_{m n} \\
B_{k q b n}^{\eta}, \quad a=b=j=p=N X \\
C_{e}^{\Gamma_{x}}=c_{0} \sum_{a=0}^{N_{x}} \sum_{m=0}^{N_{x}}(1-\mathbf{n} \cdot \mathbf{M a})_{a b}\left(\sqrt{\left(\frac{\partial x}{\partial \xi}\right)^{2}+\left(\frac{\partial y}{\partial \xi}\right)^{2}}\right)_{m n} \\
B_{j \text { pam }}^{\xi}, b=n=k=q=N Y
\end{gathered}
$$

$$
\begin{aligned}
K_{e}^{\Gamma_{y}}=c_{0}^{2} & \sum_{b=0}^{N_{y}} \sum_{n=0}^{N_{y}} \sum_{s=0}^{N_{y}}[(1-\mathbf{n} \cdot \mathbf{M a})|\mathbf{n} \times \mathbf{M a}|]_{a b} \frac{1}{|J|_{m n}} \nabla_{y, j k m n} \\
& \left(\sqrt{\left(\frac{\partial x}{\partial \eta}\right)^{2}+\left(\frac{\partial y}{\partial \eta}\right)^{2}}\right)_{r s} B_{b n q s}^{\eta}, \quad a=m=r=j=p=N X
\end{aligned}
$$

$$
\begin{aligned}
K_{e}^{\Gamma_{x}}= & c_{0}^{2} \sum_{a=0}^{N_{x}} \sum_{m=0}^{N_{x}} \sum_{r=0}^{N_{x}}[(1-\mathbf{n} \cdot \mathbf{M a})|\mathbf{n} \times \mathbf{M a}|]_{a b} \frac{1}{|J|_{m n}} \nabla_{x, j k m n} \\
& \left(\sqrt{\left(\frac{\partial x}{\partial \xi}\right)^{2}+\left(\frac{\partial y}{\partial \xi}\right)^{2}}\right)_{r s} B_{a m p r}^{i}, \quad b=n=s=k=q=N Y
\end{aligned}
$$

where $N X=\frac{(i-1)(i-3)(i-4)}{2} N_{x}, \quad N Y=-\frac{(i-1)(i-2)(i-4)}{2} N_{y}$.

The SEM was applied to the acoustic propagation problems based on linearized Euler equations [26]. It was evidenced that the implicit Newmark method in time marching presented its superiority in stability. Hence, the time marching method-the implicit Newmark method, is used in this paper.

\section{Numerical results and discussion}

The acoustic radiation in complex areas, such as multiple unequal-diameter rigid cylinders, was presented as the standard problem of CAA at the second to fourth CAA Workshops [8, 20]. Therefore, the author investigates the sound radiation near the rigid cylinder and in the trapezoid area.

\subsection{Rectangular area pierced by a circular hole}

The geometry model of the area near the cylinder can be seen as a rectangular area pierced by a circular hole, as shown in figure 5 . The rectangular domain pierced by a circular hole is divided into four sub-regions $\bar{\Omega}_{0}, \bar{\Omega}_{1}, \bar{\Omega}_{2}, \bar{\Omega}_{3}$. In each sub-region, first the arc segment and beeline segment along the $m x$-arrows are divided into average $N_{m}$ segments. Later, the evenly distributed nodes are accordingly connected into beeline segments, which will be divided into $N_{n}$ segments evenly or proportionately. Later, two cases are computed. In order to test the performance of our approximation method and the mapping method, a problem with analytical solution is computed first. Later the radiation of the rigid cylinder source with monopoles is simulated.

Case 1: The Mach number is taken as zero and $u_{\text {analyti- }}$ cal $=\sin (x) \sin (y) \cos (t)$. The computational domain is $[-\pi, \pi] \times[-\pi, \pi]$. The radius of the inside circle is 0.5 . The length unit is metre, abbreviated as " $\mathrm{m}$ ". Each sub-region is divided into $4 \times 4$ elements. The time step is taken as $\Delta t=0.001$ s. Two hundred steps have been 


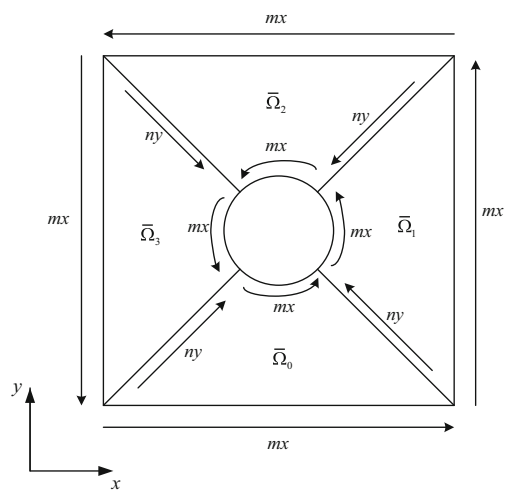

Figure 5. Rectangular area pierced by a circular hole and sketch of its mesh generation.

computed. The isoparametric SEM is very well known in and good at dealing with the problem with irregular geometries. Hence, a comparison between the SEM and the isoparametric SEM is presented in figure 6 and table 1. For comparison of the two methods, the accuracy of a numerical solution produced by approximate schemes is measured via the $L^{2}$ norm error [19]:

$$
L_{2}=\frac{1}{N}\left[\sum_{i=1}^{N}\left(u_{i}-u_{h, i}\right)\right]^{\frac{1}{2}} .
$$

where $u_{i}$ and $u_{h, i}$ are the exact and computed values of the solution, respectively; $N$ is the number of all the computed nodes.

From figure 6 it can be seen that the two methods have the same precision with the same meshes. However, with the same precision, the SEM can save almost half of the computing time compared with the isoparametric SEM
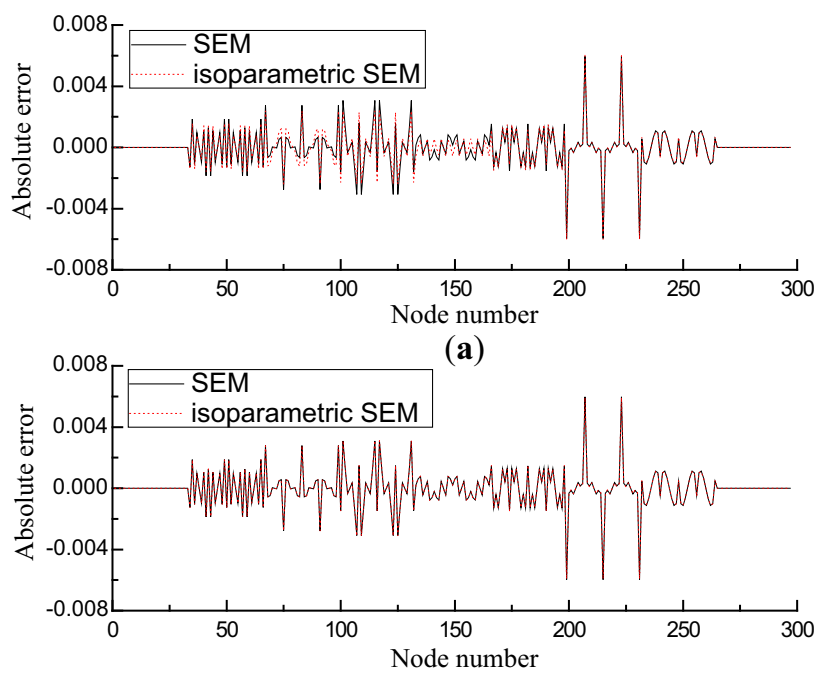

(b)

Figure 6. Compaprison of the SEM and the isoparametric SEM: (a) $t=0.1 \mathrm{~s}$ and (b) $t=0.2 \mathrm{~s}$.
Table 1. Time cost of the SEM and the isoparametric SEM for the analytical problem on the rectangular domain pierced by a circular hole.

\begin{tabular}{lrc}
\hline & \multicolumn{2}{c}{ Time cost (unit/s) } \\
\cline { 2 - 3 } Order of Chebyshev polynomials & \multicolumn{1}{c}{ SEM } & Isoparametric SEM \\
\hline$N_{x}=N_{y}=2$ & 981 & 1874 \\
$N_{x}=N_{y}=3$ & 7054 & 13,849 \\
$N_{x}=N_{y}=4$ & 81,345 & 154,131 \\
\hline
\end{tabular}

shown in table 1. Indeed, the reason is that the SEM based on the length-scale method has two superposition cycles less than the isoparametric SEM.

The $L_{2}$-error curves with different polynomial orders are shown in figure 7. Although all these results are convergent, it is clear that the accuracy is enhanced with the increasing order of the spectral approximation polynomials. The higher the spectral approximation order, the longer the consuming time and the higher the precision increases exponentially. Based on such high accuracy and absolute stability, source radiation will be computed in the next part.

Case 2: The domain is set as $[-50,50] \times[-50,50]$ and the radius of the cylinder is 8 ; the centres of the circle inside are $(0,0)$ and $(-20,0)$ respectively. $4 N_{\mathrm{m}} N_{x}$ monopole sources are distributed on the grids of the circle. The monopole source is

$$
Q(x, y, t)=\varepsilon e^{-\alpha\left[\left(x-x_{s}\right)^{2}+\left(y-y_{s}\right)^{2}\right]} \sin (\omega t)
$$

where $\varepsilon=0.5$ is the amplitude of the sound source, $\alpha=\ln 2 / \ln 22.2$ the attenuation coefficient, $\omega=20 \pi \mathrm{rad} /$ $\mathrm{s}$ the angular frequency and $\left(x_{\mathrm{s}}, y_{\mathrm{s}}\right)$ the position of the sound source.

The hard boundary conditions are given on the circle boundary and the BGH absorbing boundary conditions [2] are applied to the straight boundaries. After the study of the mesh refinement, the segments along the $m x$-arrows are evenly divided into 4 elements and the beeline segments along the $n y$-arrows are divided into 6 elements according to the ratio of 1.5. $N_{x}=N_{y}=5$ is taken in each element and the time step $\Delta t=0.0005 \mathrm{~s}$. The meshes are exhibited in figure 8 . The time evolutions of sound pressure contours generated by a monopole cylinder source in the flow with $M a=0$ and $M a=M a_{x}=0.6$ are shown, respectively, in figures 9 and 10. The acoustic wave of the monopole cylinder source spreads around the monopole point sound source. When $t=0.15 \mathrm{~s}$, the wave spreads to the boundary, and the absorption effect of the absorbing boundary conditions is so good that the wave in the corner travels normally. There are two main differences between figures 9 and 10. One is that two arc-shaped sound sources appear in figure $9 \mathrm{a}$ at the almost initial time $t=0.0005 \mathrm{~s}$. The reason is that the upwind flow increases the quality source. The 


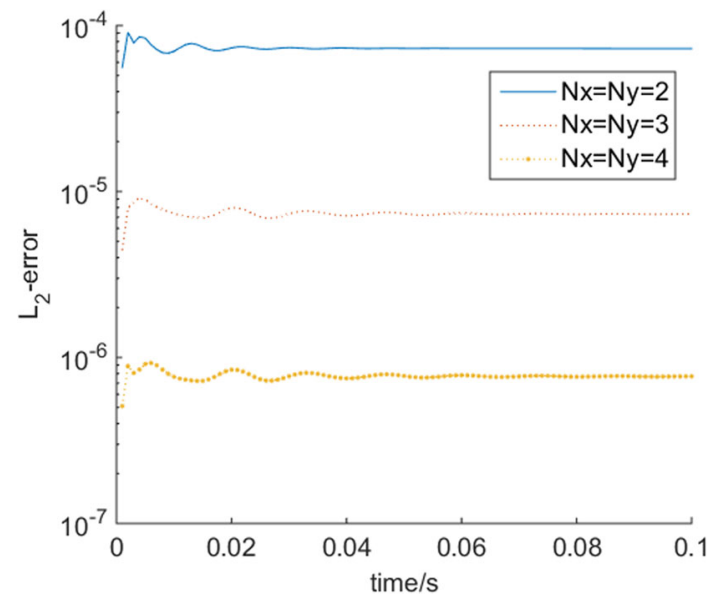

Figure 7. Convergence of SEM based on the length-scale method for analytical problem on the rectangular domain pierced by a circular hole.

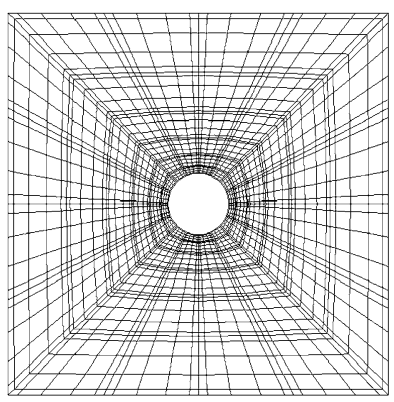

(a)

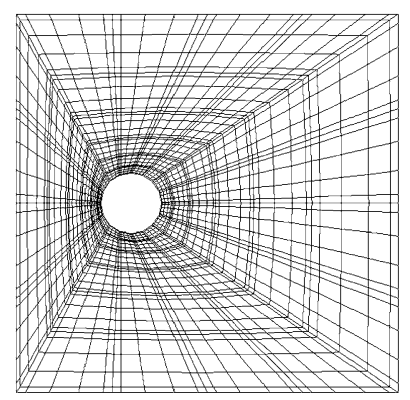

(b)
Figure 8. Grids of the rectangle pierced by a circular hole. (a) $M a=0$ (b) $M a=M a_{x}=0.6$.

effect is equivalent to introducing two ribbon sources with the phase difference of $\pi$ and the same amplitude for the monopole cylinder source. The other one is that the acoustic wave front is no longer circular, but the sound pressure contours are spread like bulbs outwards as the time increases.

\subsection{Trapezoid area}

For the trapezoid area as shown in figure 11, the edges $\Gamma_{1}$ and $\Gamma_{3}$ are evenly divided into $N_{m}$ segments first. Later the edges $\Gamma_{2}$ and $\Gamma_{4}$ are evenly divided into $N_{n}$ segments. The elements division is completed by connecting the corresponding points. There are two cases in this part, including a basic problem with analytical solution (figure 12) and the radiation of the monopole source.

Case 1: In this section, the governing equation is

$$
\frac{\partial^{2} u}{\partial t^{2}}-a^{2}\left(\frac{\partial^{2} u}{\partial x^{2}}+\frac{\partial^{2} u}{\partial y^{2}}\right)=0
$$

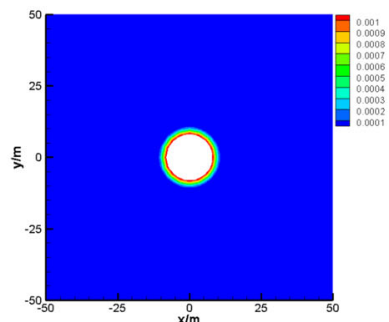

(a)

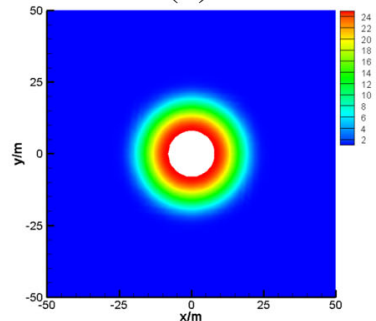

(c)

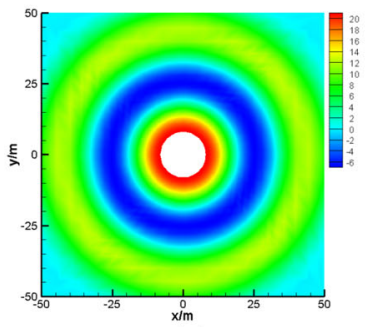

(e)

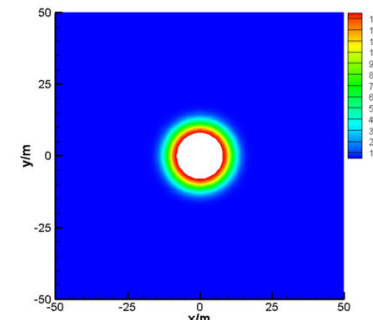

(b)

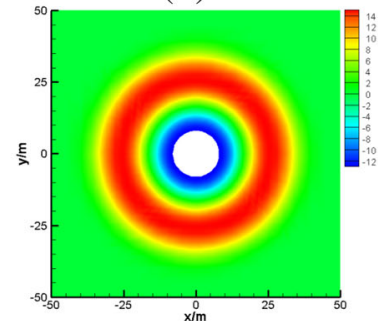

(d)

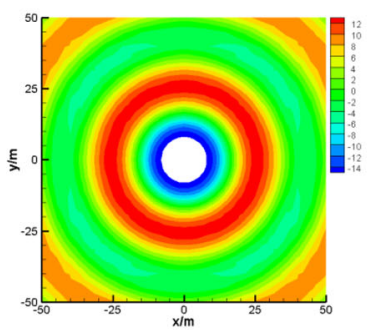

(f)
Figure 9. Radiation of cylinder source with monopoles in the rectangular domain pierced by a circular hole at $M a=0$. (a) $t=0.0005 \mathrm{~s}, \quad$ (b) $t=0.025 \mathrm{~s}, \quad$ (c) $t=0.050 \mathrm{~s}$, (d) $t=0.100 \mathrm{~s}$, (e) $t=0.150 \mathrm{~s}$, (f) $t=0.200 \mathrm{~s}$.

with the analytical solution $u=\cos (\pi a t) \sin (\pi x / \sqrt{2})$ $\sin (\pi y / \sqrt{2})+1.0$ and $a=1.0$. The domain shown in figure 11 is divided into $4 \times 4$ elements. The time step is taken as $\Delta t=0.001 \mathrm{~s}$. The numerical errors are shown in figure 13 and the time cost is shown in table 2 . They agree well with figure 7 and table 1 .

Case 2 The monopole source is the same as that in Eq. (39). The BGH absorbing boundary conditions [2] are applied on the four straight edges. After the study of the mesh refinement, $N_{m}=N_{n}=N_{x}=N_{y}=6$ are adopted and $\Delta t=0.0005 \mathrm{~s}$. The meshes are exhibited in figure 14 and 500 steps have been computed. The pressure contours are shown in figures 15 and 16.

In figure 15 , the wave travels at the same velocity in different directions with the round wave fronts. In figure $15 \mathrm{a}-\mathrm{d}$, the wave has not spread to the boundary. At $t=0.15 \mathrm{~s}$, the wave front arrives at the boundaries. At $t=0.20 \mathrm{~s}$, the wave has travelled to the corners. It can be seen that there is no obvious distortion in the contours not only on the hypotenuse but also in the corner. 


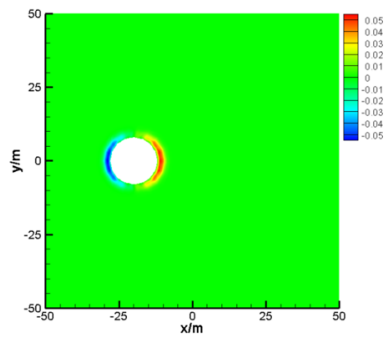

(a)

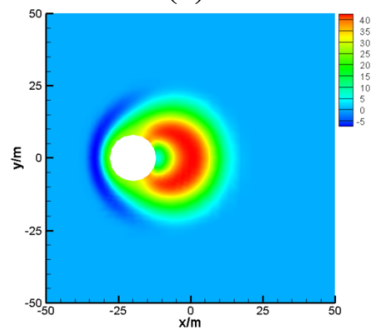

(c)

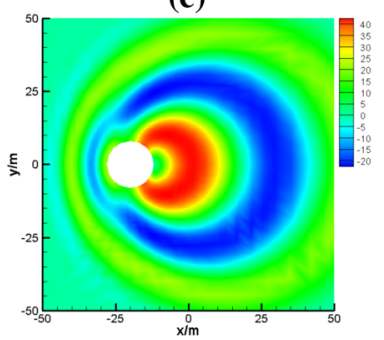

(e)

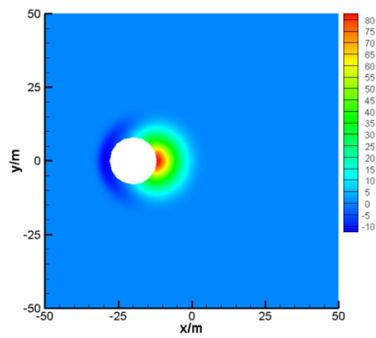

(b)

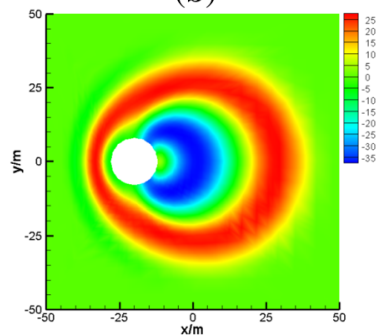

(d)

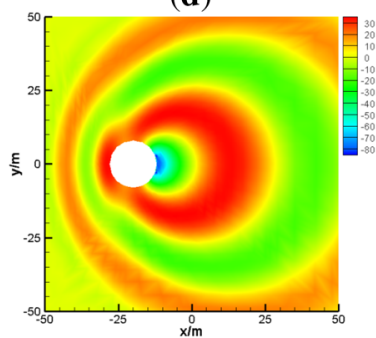

(f)
Figure 10. Radiation of cylinder source with monopoles in the rectangular domain pierced by a circular hole at $M a=M a_{x}=0.6$. (a) $t=0.0005 \mathrm{~s}$, (b) $t=0.025 \mathrm{~s}$, (c) $t=0.050 \mathrm{~s}$, (d) $t=0.100 \mathrm{~s}$, (e) $t=0.150 \mathrm{~s}$, (f) $t=0.200 \mathrm{~s}$.

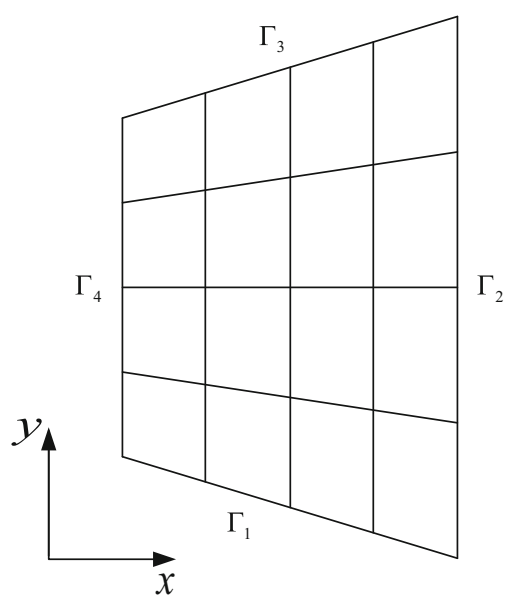

Figure 11. Trapezoid area and its sketch of mesh generation.

Figure 16a shows that a dipole appears at the almost initial time. The flow on the monopole source is equivalent to adding another monopole. However, it does not spread as a dipole. At $t=0.015 \mathrm{~s}$, the upstream wave has bigger

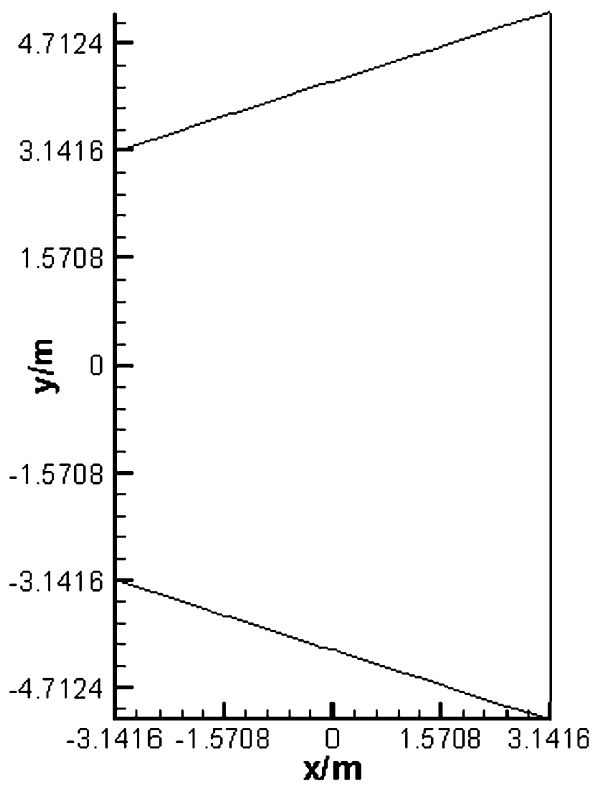

Figure 12. Trapezoid domain for an analytical solution.

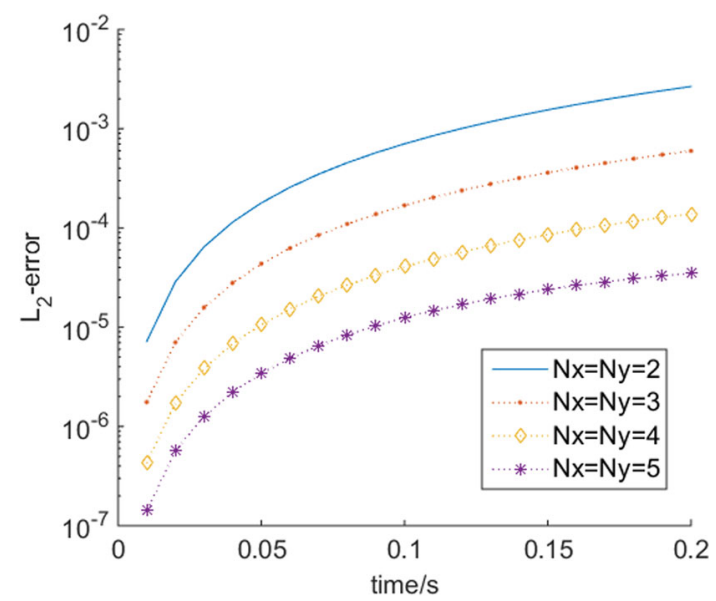

Figure 13. Convergence of SEM integrated with the length-scale method for the analytical problem in the trapezoid area.

amplitude while the downstream wave has higher velocity. The wave front is circular as the time increases. Simultaneously, perfect absorbing effect is found except near the hypotenuse boundaries shown in figure 16f for the reason that the pressure wave could not be absorbed completely by BGH absorbing boundary conditions.

The monopole source radiation on the rectangular domain has been computed by Zhang et al [25]. Some comparisons between the results in this paper and in reference [25] are given in the following by means of profiles on the horizontal midline $(y=0)$. It is necessary to illustrate in advance that the parameters and the position of monopole source in this paper are the same as those in reference [25]. The profiles at $M a=0$ and 
Table 2. Time cost of SEM for analytical problem in the trapezoid area.

\begin{tabular}{lc}
\hline Order of Chebyshev polynomials & Time cost (unit/s) \\
\hline$N_{x}=N_{y}=2$ & 71 \\
$N_{x}=N_{y}=3$ & 379 \\
$N_{x}=N_{y}=4$ & 1521 \\
$N_{x}=N_{y}=5$ & 6214 \\
\hline
\end{tabular}

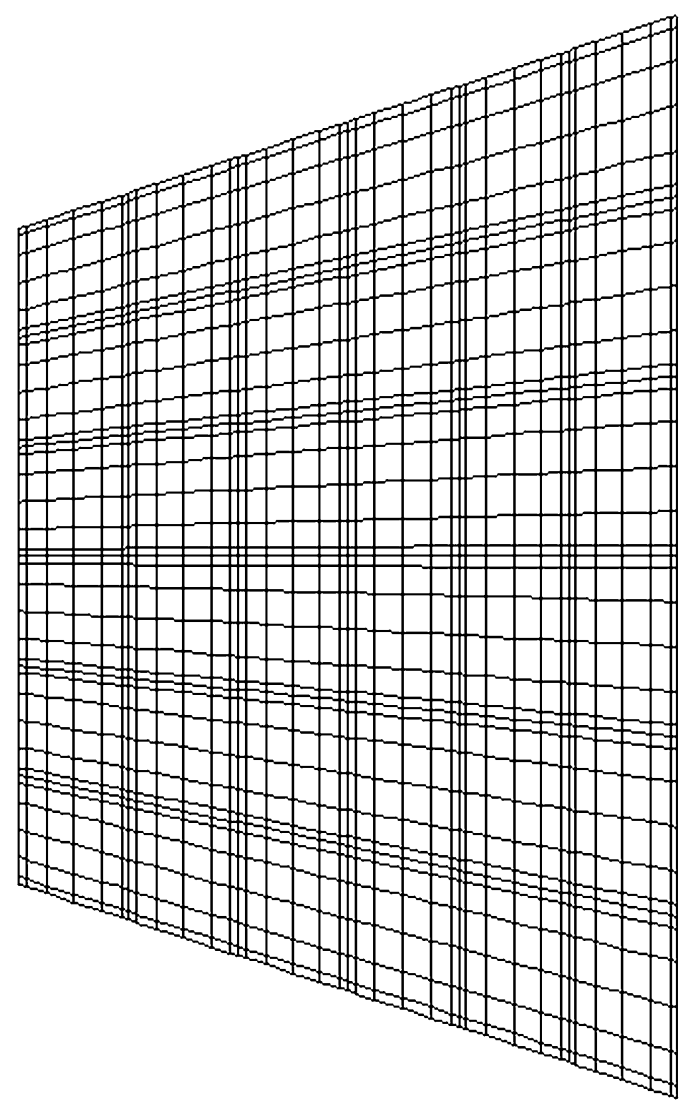

Figure 14. Grids of the trapezoid domain.

$M a=M a_{x}=0.6$ are shown in figures 17 and 18, respectively. As can be seen clearly, the two curves are almost the same except in the vicinity of extreme points and endpoints at both $t=0.1 \mathrm{~s}$ and $t=0.2 \mathrm{~s}$. The reason is probably that there are some numerical reflections induced by the nonreflecting boundaries. An affirmative conclusion can be drawn that the two curves agree well with each other. All these verify that the results in this paper are consistent with the ones in [25]. This indicates that the length-scale method is reliable in dealing with irregular areas from the view of the numerical analysis.

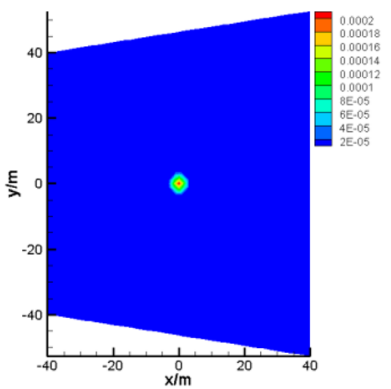

(a)

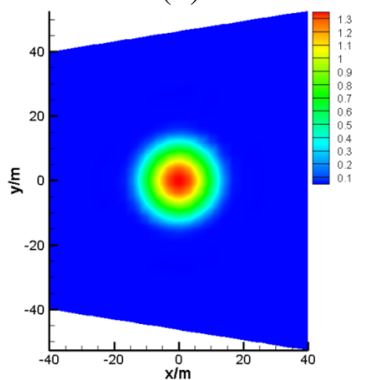

(c)

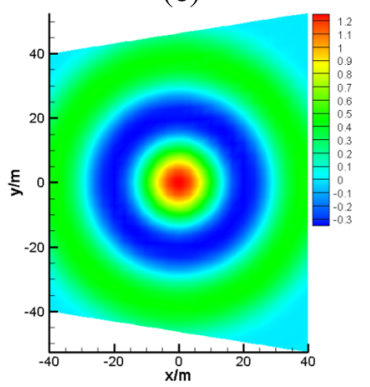

(e)

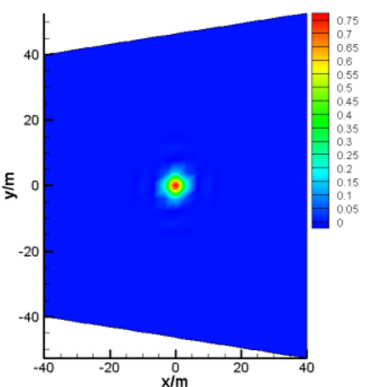

(b)

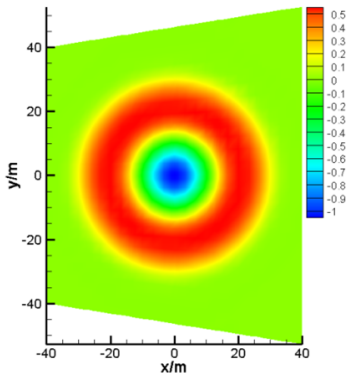

(d)

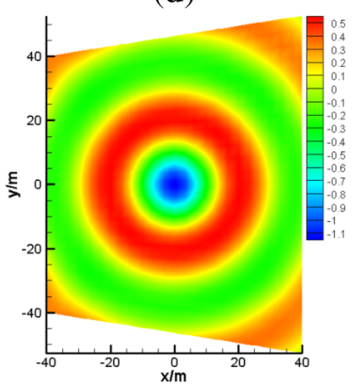

(f)
Figure 15. Monopole sources radiation in the trapezoid domain at $M a=0$. (a) $t=0.0005 \mathrm{~s}$, (b) $t=0.015 \mathrm{~s}$, (c) $t=0.050 \mathrm{~s}$, (d) $t=0.100 \mathrm{~s}$, (e) $t=0.150 \mathrm{~s}$, (f) $t=0.200 \mathrm{~s}$.

\section{Conclusions}

SEM holds a great position in numerical computations; however, it cannot deal with irregular domains efficiently and economically. Therefore, the length-scale method is proposed to map the irregular domains to the standard one. Using the length-scale method on irregular domains, the SEM can have the same accuracy as the isoparametric SEM and save half of the computing time as well. Additionally, it is demonstrated that the SEM integrated with the lengthscale method has the ability to compute the acoustic problems with irregular domains. In general, the lengthscale method can be applied to problems on irregular domains simply and reliably. Hence, it will further be used in various engineering problems, such as the external and internal flow, the biomedical engineering, the cardiac electrophysiology, etc. 


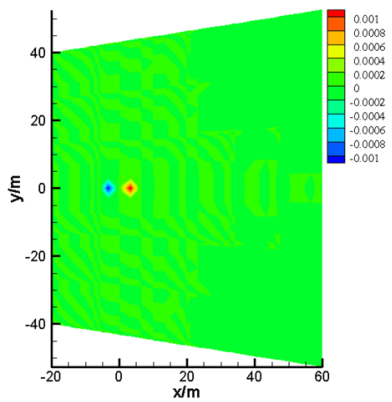

(a)

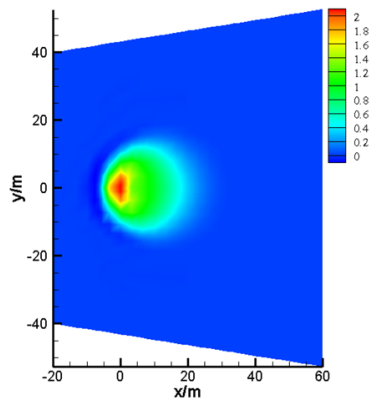

(c)

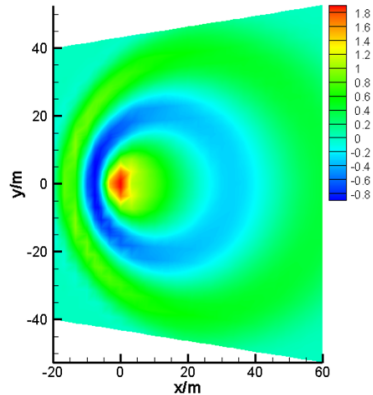

(e)

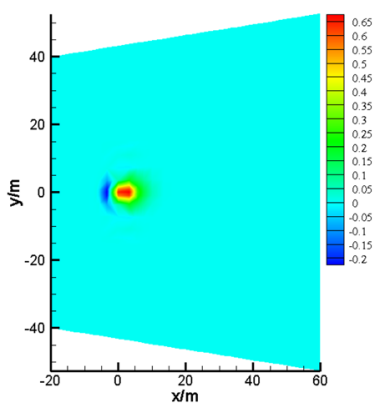

(b)

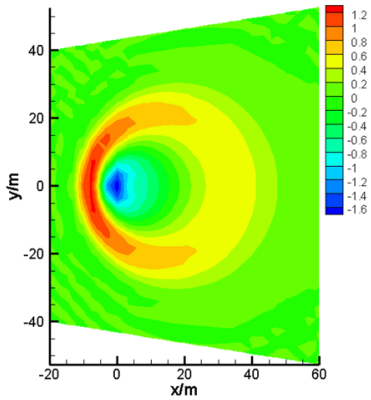

(d)

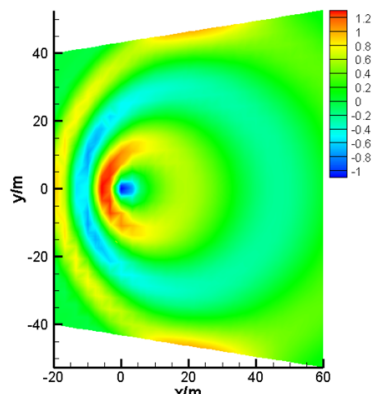

(f)
Figure 16. Monopole sources radiation on the trapezoid domain at $M a=M a_{x}=0.6$. (a) $t=0.0005 \mathrm{~s}, \quad$ (b) $t=0.015 \mathrm{~s}$, (c) $t=0.050 \mathrm{~s}$, (d) $t=0.100 \mathrm{~s}$, (e) $t=0.150 \mathrm{~s}$, (f) $t=0.200 \mathrm{~s}$.

\section{Acknowledgements}

The authors would like to acknowledge the support by the National Fundamental Research Program of China (No. 2012CB026004).

\section{Appendix A}

There are three equations given in section 2 :

$$
\begin{gathered}
\frac{\overparen{P_{4} A}}{\overparen{P_{4} P_{3}}}=\frac{\xi+1}{2} \\
\frac{P_{1} B}{P_{1} P_{2}}=\frac{\xi+1}{2}
\end{gathered}
$$

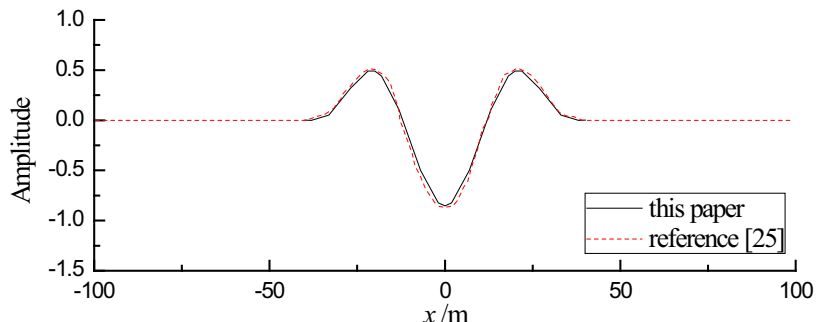

(a)

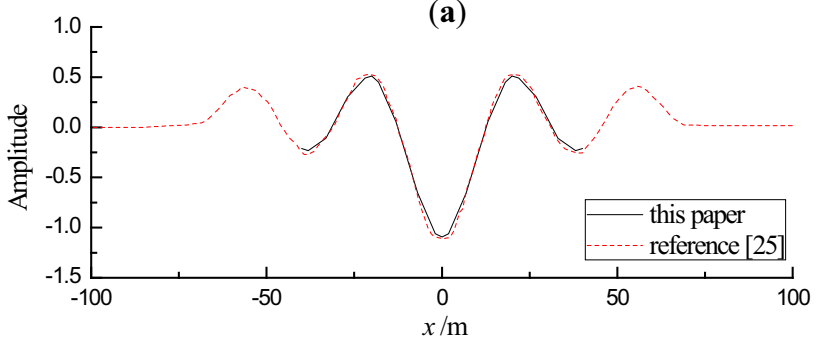

(b)

Figure 17. Comparisons between the results in this paper and in reference [25] at $M a=0$ : (a) $t=0.1 \mathrm{~s}$ and (b) $t=0.2 \mathrm{~s}$.
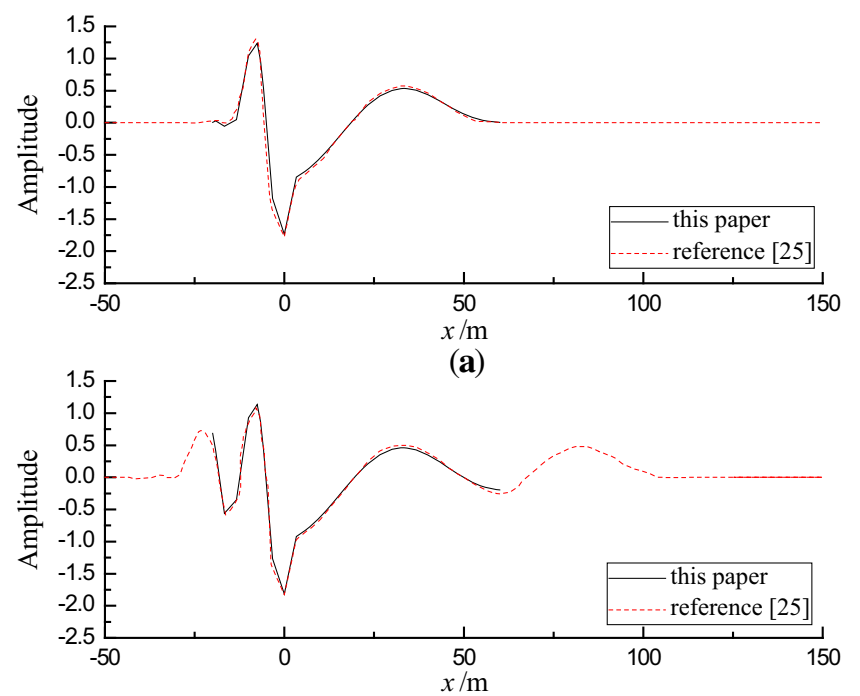

(b)

Figure 18. Comparisons between the results in this paper and in reference [25] at $M a=M a_{x}=0.6:(\mathbf{a}) t=0.1 \mathrm{~s}$ and (b) $t=0.2 \mathrm{~s}$.

$$
\frac{B O}{B A}=\frac{\eta+1}{2}
$$

According to the geometrical relations, the expressions on coordinate system for Eq. (A.1) is presented as follows:

$$
\overparen{P_{4} A}=R \phi
$$

in which $\phi=c_{1}-\theta, \theta=\angle A \Theta C$ are in radians. From Eq. (A.4), $\theta$ can be obtained as

$$
\theta=c_{1}-\frac{\xi+1}{2}\left(c_{1}-c_{2}\right)
$$


Also

$$
\begin{aligned}
& x_{A}=x_{\Theta}+R \cos \theta, \\
& y_{A}=y_{\Theta}-R \sin \theta .
\end{aligned}
$$

At the same time, the expressions on coordinate system for Eq. (A.2) are

$$
\begin{aligned}
& \frac{x_{B}-x_{1}}{x_{2}-x_{1}}=\frac{\xi+1}{2}, \\
& \frac{y_{B}-y_{1}}{y_{2}-y_{1}}=\frac{1-\xi}{2} .
\end{aligned}
$$

Through some easy algebraic transformations, $x_{B}$ and $y_{B}$ can be obtained from Eqs. (A.8) and (A.9) as follows:

$$
\begin{aligned}
& x_{B}=\frac{\xi+1}{2}\left(x_{2}-x_{1}\right)+x_{1}, \\
& y_{B}=\frac{1-\xi}{2}\left(y_{2}-y_{1}\right)+y_{1} .
\end{aligned}
$$

Besides, the expressions on coordinate system for Eq. (A.3) are

$$
\begin{aligned}
& \frac{x_{O}-x_{B}}{x_{A}-x_{B}}=\frac{1-\eta}{2}, \\
& \frac{y_{O}-y_{B}}{y_{A}-y_{B}}=\frac{1+\eta}{2} .
\end{aligned}
$$

Thus, $x_{O}$ and $y_{O}$ can be obtained from Eqs. (A.12) and (A.13) as follows:

$$
\begin{aligned}
& x_{O}=\frac{1-\eta}{2}\left(x_{A}-x_{B}\right)+x_{B}, \\
& y_{O}=\frac{1+\eta}{2}\left(y_{A}-y_{B}\right)+y_{B} .
\end{aligned}
$$

Finally, the expressions for $x_{O}, y_{O}$ can be obtained by plugging Eqs. (A.5)-(A.7), (A.10) and (A.11) into Eqs. (A.14) and (A.15). Indeed, the expressions are the mapping relationships from physical space to the standard space.

\section{References}

[1] Auteri F et al 2001 Incompressible Navier-Stokes solutions by a triangular spectral/p element projection method. Comput. Methods Appl. Mech. Eng. 190: 6927-6945

[2] Bécache E et al 2010 High-order absorbing boundary conditions for anisotropic and convective wave equations. $J$. Comput. Phys. 229(4): 1099-1129

[3] Chuang J J and Yang D C H 2004 A boundary-blending method for the parametrization of 2D surfaces with highly irregular boundaries. J. Mech. Des. 126(2): 327-335
[4] Du J and Fogelson A L 2011 A Cartesian grid method for two-phase gel dynamics on an irregular domain. Int. J. Numer. Methods Fluids 67(12): 1799-1817

[5] Giraldo F X and Taylor M A 2006 A diagonal mass matrix triangular spectral element method based on cubature points. J. Eng. Math. 56(3): 307-322

[6] Giraldo F X and Warburton T 2005 A nodal triangle-based spectral element method for the shallow water equations on the sphere. J. Comput. Phys. 207(1): 129-150

[7] Goldstein M E 1974 Unified approach to aerodynamic sound generation in the presence of sound. J. Acoust. Soc. Am. 56(5): 499-509

[8] Kim J W and Lee D J 2000 Fourth computational aeroacoustics (CAA) workshop on benchmark problems. In: Proceedings of the NASA Conference, Cleveland

[9] Kosec G 2016 A local numerical solution of a fluid-flow problem on an irregular domain. Adv. Eng. Softw. 1-9

[10] Kumar P and Ik K K 2015 Hydrodynamic modeling of moored ship motion in an irregular domain. Procedia Eng. 127: 598-604

[11] Mengaldo G et al 2015 Dealiasing techniques for high-order spectral element methods on regular and irregular grids. $J$. Comput. Phys. 299: 56-81

[12] Mercerat E D et al 2006 Triangular spectral element simulation of two-dimensional elastic wave propagation using unstructured triangular grids. Geophys. J. Int. 166(2): 679-698

[13] Moxey D et al 2015 An isoparametric approach to high-order curvilinear boundary-layer meshing. Comput. Methods Appl. Mech. Eng. 283: 636-650

[14] Pasquetti R 2016 Comparison of some isoparametric mappings for curved triangular spectral elements. J. Comput. Phys. 316: 573-577

[15] Pasquetti R and Rapetti F 2004 Spectral element methods on triangles and quadrilaterals: comparisons and applications. $J$. Comput. Phys. 198(1): 349-362

[16] Patera A T 1984 A spectral element method for fluid dynamics: laminar flow in a channel expansion. J. Comput. Phys. 54: 468-488

[17] Pontaza J P 2007 A spectral element least-squares formulation for incompressible Navier-Stokes flows using triangular nodal elements. J. Comput. Phys. 221(2): 649-665

[18] Pozrikidis C 2014 The finite element method in two dimensions. In: Introduction to Finite and Spectral Element Methods Using MATLAB. Hoboken: CRC Press

[19] Shao W et al 2012 Chebyshev tau meshless method based on the integration-differentiation for Biharmonic-type equations on irregular domain. Eng. Anal. Boundary Elem. 36(12): $1787-1798$

[20] Tam C K W and Hardin J C 1996 Second computational aeroacoustic (CAA) workshop on benchmark problems. In: Proceedings of the NASA Conference, Tallahassee

[21] Theillard M et al 2013 A second-order sharp numerical method for solving the linear elasticity equations on irregular domains and adaptive grids-application to shape optimization. J. Comput. Phys. 233: 430-448

[22] Vosse F N V D and Minev P D 1996 Spectral element methods: theory and applications. Eindhoven: Eindhoven University of Technology

[23] Wan M et al 2005 Numerical prediction of static form errors in peripheral milling of thin-walled workpieces with irregular meshes. J. Manuf. Sci. Eng. 127(1): 13-22 
[24] Wu X and Han G 2011 Direct expansion method of boundary condition for solving 3D elliptic equations with small parameters in the irregular domain. Comput. Math. Appl. 61(10): 2971-2980

[25] Zhang R X et al 2009 Spectral elements method for acoustic propagation problems based on linearized euler equations. $J$. Comput. Acoust. 17(4): 383-402
[26] Zhu C Y et al 2011 Implicit Chebyshev spectral element method for acoustics wave equations. Finite Elem. Anal. Des. 47(2): 184-194 